\title{
Optimal Schedule of Distributed Generation in a Grid-Tied Micro-grid via Minimum Incremental Cost Approach
}

\author{
Wei-Tzer Huang, Kai-Chao Yao, and Chun-Ching Wu
}

\begin{abstract}
This paper proposed a simple and efficient approach, minimum incremental cost approach (MICA), for the optimal schedule in a grid-tied micro-grid (MG) with various types of distributed generation (DG). The fuel costs generated by these DGs are determined using quadratic and linear functions dependent on the types of DGs. The proposed approach, MICA, is able to handle several inequality constraints without introducing any multipliers and furthermore it can solve the non-derivative problems or the fuel cost functions being much more complicated. Accordingly, the MICA is proposed for determining the optimal schedule of MGs with various types of DG to minimize generation costs under grid-tied operation. Results demonstrate that the proposed MICA is a simple approach to determining the optimal schedule in MGs with various types of DG.
\end{abstract}

Index Terms-Minimum incremental cost approach, micro-grid, distributed energy resources, distributed generation, optimal schedule.

\section{INTRODUCTION}

For reducing the emission of greenhouse gases, instead of fossil energy, renewable energy should be developed. Electricity generation from large, fossil fuel-based thermal power plants should first be reduced. A suitable alternative is to gradually increase electricity generation from clean and renewable DG technologies, which are interconnected with distribution systems. It is expected that lots of distributed energy resources (DERs), such as DG and distributed storage (DS) systems will interconnected with traditional electrical power distribution systems in the near future. The DG penetration levels of existing systems improve yearly because of sufficiently mature technological development and research. A MG is the part of a distribution system that includes DERs and loads with a single point of common coupling (PCC) [1], [2]. The MG can be operated in grid-tied and islanded modes. The use of MGs is the new development trend in power systems; hence, investigating the structure of the system, related operating control technology, and scheduling management is necessary.

Related research approaches to the optimal schedule, unit commitment, and online management of power systems; these approaches include Lagrange multiplier algorithms, dynamic

Manuscript received September 3, 2014; revised January 10, 2015. This work was supported by the National Science Council of Taiwan, R.O.C., for the financial support under Grant No. NSC 102-2511-S-018-016.

The authors are with the Department of Industrial Education and Technology, National Changhua University of Education, Changhua 50007, Taiwan (e-mail: vichuang@cc.ncue.edu.tw, kcyao@cc.ncue.edu.tw, D0231007@cc.ncue.edu.tw). programming algorithms, evolution algorithms [3]-[4], direct search algorithms [5]-[7], and particle swarm optimization [8]. These approaches have their advantages and disadvantages, where the direct search algorithms use only function values and require only a numerical value without any knowledge of the internal structure of a problem. Like direct search method, the MICA is easily adapted to nonlinear, non-convex, non-differentiable, or disconnected problems, making it highly suitable for solving the optimal schedule problem in MGs with various DG types that are composed of different operation cost functions. Based on the optimization techniques mentioned above, this paper proposed simple and efficient MICA for solving optimal schedule problem. We simulated the optimal schedule of DG, considering the minimization of fuel costs under grid-tied condition.

\section{DESCRIPTION OF THE PROBLEM}

Optimal schedule in MGs is intended for the effective and safe management of various DG systems and controllable loads. Given this purpose, we used a Taipower $11.4 \mathrm{kV}$ distribution system to plan and design a MG that is composed of two primary distribution feeders. The proposed MICA is used to solve the optimal schedule problem under grid-tied condition. The detailed descriptions of the MG system structure and the related DG parameters are discussed in the succeeding subsections.

\section{A. Architecture of the $M G$}

Fig. 1 shows a typical Taipower distribution system, which comprises a $25 \mathrm{MVA}, 69 \mathrm{kV} / 11.4 \mathrm{kV}, 60 \mathrm{~Hz}$ distribution transformer and 5 primary distribution feeders. The $11.4 \mathrm{kV}$ medium-voltage MG is part of the distribution system. Feeders F\#1 and F\#2 can be regarded as a two-feeder MG (F\#1\&2 MG) with various DG types and lump-sum loads. The remaining feeders, F\#3-F\#5, are traditional feeders without DG, and can be considered having lump-sum loads.

Feeder F\#1 consists of the following DG units: two gas turbine generators, two fuel cell generation systems, and a photovoltaic generation system. The equivalent lump-sum load types of feeder F\#1 are residential, office, and commercial. Feeder F\#2 consists of the following DG units: two diesel engine generators and a wind turbine generator. The equivalent lump-sum load types of feeder F\#2 are residential and industrial. The above-mentioned DG units are all interconnected with primary feeders by power electronic converters, inverters, and isolated power transformers. A static switch is installed in the PCC of the MGs for grid-tied and islanded operating conditions. 


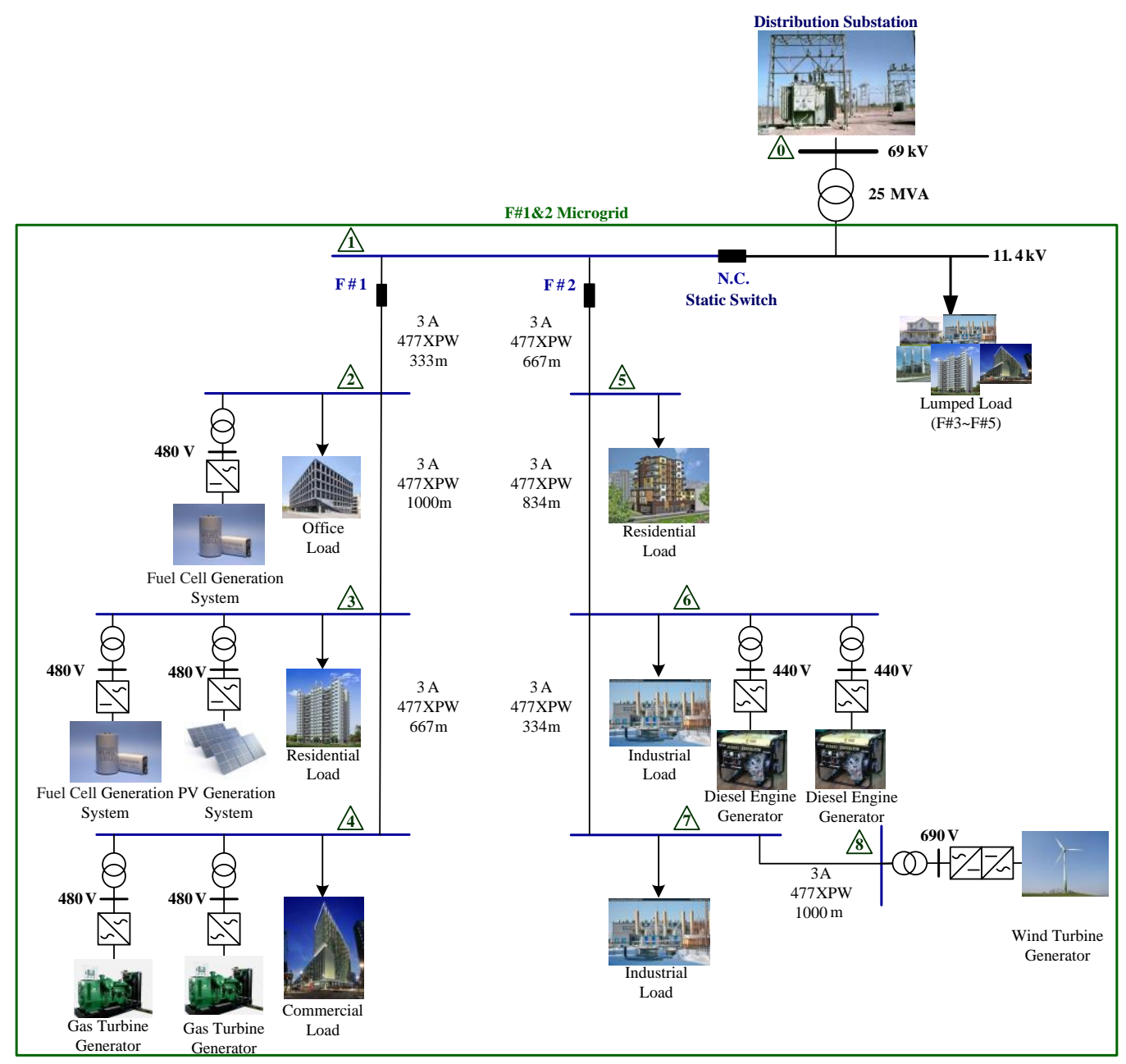

Fig. 1. System architecture of an $11.4 \mathrm{kV}$ medium-voltage MG.

\section{B. Operating Conditions}

There are four operating conditions of the MGs, as show as follows:

1) Steady-state grid-tied operation;

2) Steady-state islanded operation under planned and unexpected situations;

3) Transient operation from grid-tied to islanded conditions;

4) Transient operation from islanded to grid-tied conditions.

In optimal schedule problem, only steady-state grid-tied and islanded operating conditions were considered. According to system structure, the available power output of DG units, and associated load demands, the proposed MICA was used to solve the optimal schedule problem of DG in the MG under the aforementioned operating conditions. This work is similar to those on the economic dispatch or unit commitment in traditional power systems that consider coordination between available power and renewable energy units.

In grid-tied operation, through a static switch, the F\#1\&2 MG is interconnected with the upper power grid in the PCC as shown in Fig. 1. Under the grid-tied mode, the static switch is normally closed. Based on the minimization of fuel costs and power balance principle, the load demand in an $\mathrm{MG}$ is supplied by DG units and the purchased power from an upper power grid. Moreover, if the power supplied from DG units is greater than the load demand, the power will reverse to upper power grid through the PCC.

\section{Parameters of the DGs}

The best strategies for system operation are economical and reliable approaches, as well as those that present low emissions. The optimal schedule problem of DG units in MGs is minimizing generation cost and reducing environmental pollution. The generation costs of an MG include fuel and emission costs, operation cost, maintenance cost, and purchasing or selling cost. To accurately calculate the generation cost of the MG in this paper, we considered the available power output of renewable energy DG units and fuel cost of non-renewable energy DG units.

\section{1) Available power output of renewable energy DGs}

a) Wind turbine generator

The available power output of a wind turbine generator is a function of wind speed. Equation (1) shows the model used to calculate the output power generated by the wind turbine generator [9], [10]:

$$
\left\{\begin{array}{lr}
P_{w t}=0, & V<V_{c i} \\
P_{w t}=a \cdot V^{3}+a \cdot V^{2}+c \cdot V+d, & V_{c i} \leq V<V_{r} \\
P_{w t}=P_{w t, r}, & V_{r} \leq V<V_{c o} \\
P_{w t}=0, & V \geq V_{c o}
\end{array}\right.
$$

where $P_{w t, r}$ is the rated power. $V, V_{r}, V_{c i}$, and $V_{c o}$ are the actual, 
rated, cut-in, and cut-out wind speeds, respectively. A GE-1.5xle wind turbine generator was used. The parameters in the model are as follows: $a=-2.608, b=63.201, c=293.3$, $d=374.23, P_{w t, r}=1,500 \mathrm{~kW}, V_{c i}=3.5 \mathrm{~m} / \mathrm{s}, V_{r}=20 \mathrm{~m} / \mathrm{s}$, and $V_{c o}=12.5 \mathrm{~m} / \mathrm{s}$. Using equation (1) and the measured wind speed curve in Fig. 2, we can obtain the power output curve of the GE-1.5xle wind turbine generator.

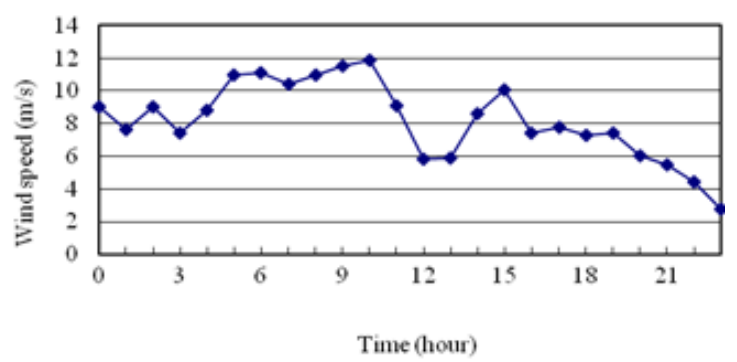

Fig. 2. Measured wind speed curve.

\section{b) Photovoltaic generation system}

The power output of the photovoltaic module can be calculated using equation (2) with the measured solar irradiation $\left(\mathrm{W} / \mathrm{m}^{2}\right)$ and ambient temperature $\left({ }^{\circ} \mathrm{C}\right)[11]$ :

$$
P_{p v}=P_{s t c} \frac{G_{\text {ing }}}{G_{s t c}}\left[1+k\left(T_{c}-T_{r}\right)\right]
$$

where $P_{p v}$ is the output power of the module at irradiance $G_{i n g}$; $P_{\text {stc }}$ denotes the maximum power at the standard test condition; $G_{\text {ing }}$ is the incident irradiance; $G_{s t c}$ represents the irradiance at the standard test condition $\left(1000 \mathrm{~W} / \mathrm{m}^{2}\right) ; k$ is the temperature coefficient of power; $T_{c}$ denotes the cell temperature; and $T_{r}$ is the reference temperature. A KYOCERA-2P 210Wp photovoltaic module was used. According to equation (2), the solar irradiation and ambient temperature were measured in Fig. 3. The power output curve of the photovoltaic generation system was also obtained.

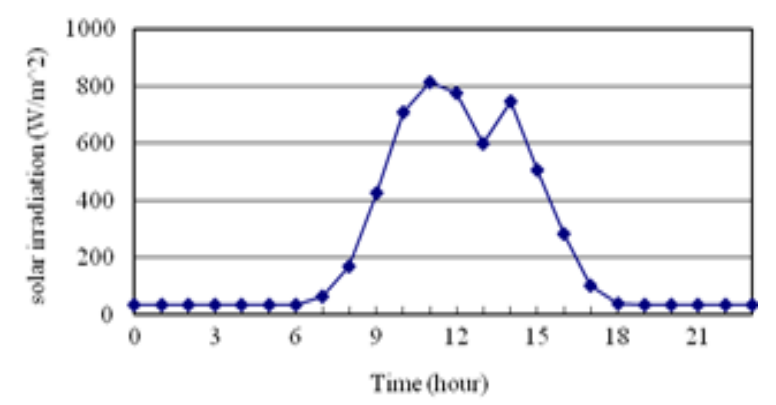

Fig. 3. Measured solar irradiation curve.

\section{2) Fuel costs of non-renewable energy DG}

\section{a) Diesel engine generator}

The fuel cost of the diesel engine generator can be expressed as a quadratic polynomial function of its real power output, as shown in equation (3) [12]:

$$
C_{D E}=\alpha+\beta \cdot P_{D E}+\gamma \cdot P_{D E}^{2}
$$

where $\alpha, \beta$, and $\gamma$ are the generator coefficients that can be obtained from the data sheet of the manufacturer. $P_{D E}$ is the power output of the diesel engine generator in $\mathrm{kW}$, and is assumed to be known. The derivative of equation (3) with respect to $P_{D E}$ (the incremental fuel cost of the diesel engine unit) can be derived and expressed as equation (4). The incremental cost (IC) under different power generations can be calculated as follows:

$$
I C_{D E}=\frac{d C_{D E}}{d P_{D E}}=\beta+2 \gamma \cdot P_{D E}
$$

\section{b) Fuel cell generation system}

Four major types of commercialized operating fuel cells currently exist: molten carbonate fuel cell (MCFC), solid oxide fuel cell (SOFC), proton exchange membrane fuel cell (PEMFC), and phosphoric acid fuel cell (PAFC). The fuel input of MCFC and SOFC is natural gas, and their rated capacities are relatively larger than that of PEMFC. Therefore, the MCFC and SOFC are more suitable for medium-voltage MGs. These two types of fuel cells were used in this study. The fuel cost of the fuel cell generator system is shown in equation (5) [13]:

$$
C_{F C}=C_{n l} \sum_{J} \frac{P_{J}}{\eta_{J}}
$$

where $C_{n l}$ is the natural gas price, $P_{J}$ denotes the net electrical power generated at interval $J$, and $\eta_{J}$ represents the fuel cell efficiency at interval $J$. The derivative of equation (5) with respect to variable $P_{J}$ (the IC of the fuel cell generator unit at interval $J$ ) is derived thus:

$$
I C_{F C}=\frac{d C_{F C}}{d P_{J}}=\frac{C_{n l}}{\eta_{J}}
$$

\section{c) Gas turbine generator}

Similar to the fuel cost of the diesel engine generator, which of the gas turbine generator can be expressed as a quadratic polynomial function of its real power output:

$$
C_{G T}=\alpha+\beta \cdot P_{G T}+\gamma \cdot P_{G T}^{2}
$$

The derivative of equation (7) with respect to variable $P_{G T}$ (the IC of the gas turbine unit) can be derived thus:

$$
I C_{G T}=\frac{d C_{G T}}{d P_{G T}}=\beta+2 \gamma \cdot P_{G T}
$$

\section{MICA FOR OPTIMAL SCHEDULE}

Many algorithms and techniques, such as mesh adaptive direct search, sequential quadratic programming, genetic algorithms, and game theory, have been used to solve the optimal schedule problems of power systems. This paper proposes the MICA to solve the optimal schedule problem of a medium-voltage MG. The concept of the proposed MICA is according to the unit with minimum incremental cost is chosen to increase its output, until the power balance. Therefore, the basic principle of the proposed algorithm comes from the combinations of the conventional Lagrangian Relaxation method and dynamic programming method. The proposed method has no restrictions on the cost functions of distributed generations, which included distributed generators and power purchased/sold from power grid in MG, and performs a direct search of solution space. This algorithm 
begins with an initial feasible solution and search for the optimal solution along a feasible path at all time. Units in $\mathrm{MG}$ without violating the upper or lower limits are to increase or decrease their generations by a predetermined step $P_{D}$ for computing their incremental cost (IC) and decrement cost (DC).

In this paper, the power output limits of the DG units of the MG can be derived after studying the power output models of renewable DG, the cost functions of non-renewable DG, the objective function that considers fuel cost minimization, and the constraints of power balance.

Step 1: input data: load demand, maximum demand contract, purchased/sold electricity cost, cost function of DGs, and generation limits of DGs.

Step 2: set up the computation step $\Delta \mathrm{P}$, and compute the initial values of the IC for each DG using (4), (6) and (8).

\section{$\downarrow$}

Step 3: read the power demand $P_{D}$, and calculate the summation of minimum output for each DG and minimum purchased electricity from upstream power grid(only under gridtied operation condition), then calculate the mismatch of power balance $(\varepsilon)$ using (10), if it is power balance, then stop the iteration procedure, and go to step 7 .

Step 4: if $\varepsilon$ is negative, it means the power demand is greater than power output, the DG without violating the upper limits is chosen to increase its output by computation step $\triangle P$ (the upstream power grid is included in grid-tied mode). Compute new value of IC for the dispatched DG, and calculate $\varepsilon$, if it is power balance, then it is convergence, and go to step 7 .

Step 5: if $\varepsilon$ is positive, it means the power demand is less than power output, the DG without violating the lower limits is chosen to decrease its output by computation step $\Delta \mathrm{P}$ (the upstream power grid is included in grid-tied mode). Restore the previous value of IC for the dispatched DG, and calculate $\varepsilon$, if it is power balance, then it is convergence, and go to step 7 .

Step 6: calculate the output of each DG and the total $\operatorname{cost} C_{T}$ using (9)

$\vee$

Step 7: stop calculations and output hourly optimal dispatch results.

Fig. 4. The procedure of the proposed MICA.

The objective function for the optimal schedule problem at time $t$ can be expressed as follows:

$$
\text { Minimize } C_{T}(t)=\sum_{t=1}^{N}\left\{\begin{array}{l}
C_{i}\left(P_{D G_{i}}(t)\right) \\
+C_{\text {Purchased }}\left(P_{\text {Purchased }}(t)\right) \\
-C_{\text {Sold }}\left(P_{\text {Sold }}(t)\right)
\end{array}\right\}
$$

Subject to

$$
\left\{\begin{array}{l}
P_{D G_{i}}(t)+P_{\text {Purchased }}(t) \\
+P_{w t}(t)+P_{p v}(t)
\end{array}\right\}=P_{D}(t)+P_{\text {Sold }}(t)
$$

And

$$
P_{D G_{i}, \min } \leq P_{D G_{i}}(t) \leq P_{D G_{i}, \text { max }} \text { for } i=1, \ldots, N
$$

Defining that the power mismatch is

$$
\varepsilon=P_{D G_{i}}(t)+P_{\text {Purchased }}(t)+P_{w t}(t)+P_{p v}(t)-P_{D}(t)-P_{\text {Sold }}(t)
$$

where $C_{i}(t)$ is the cost function of $i$ th DGs at time $t ; P_{D G_{i}}(t)$ denotes the power generation of $i$ th DGs at time $t ; P_{D G_{i} \text {,min }}$ is the lower generation limit of $i$ th DGs; $P_{D G_{i} \text {,max }}$ represents the upper generation limit of $i$ th DGs; $P_{D}(t)$ represents the total load demand of the MG at time $t$. Additionally, $C_{\text {Purchased }}(t)$ is the electricity purchased cost at time $t ; C_{\text {Sold }}(t)$ is the electricity sold cost at time $t ; P_{\text {Purchased }}(t)$ represents the power purchased from the upper utility power grid; $P_{\text {Sold }}(t)$

\begin{tabular}{|c|c|c|c|c|}
\hline Parameters & $\begin{array}{c}\text { Rated } \\
\text { Capacity }\end{array}$ & \multicolumn{3}{|c|}{ Generating Cost of Coefficient } \\
\hline DG & $(\mathrm{kW})$ & $\alpha(\$ / h)$ & $\beta(\$ / \mathrm{kWh})$ & $\gamma\left(\$ / \mathrm{kWh}^{2}\right)$ \\
\hline $\begin{array}{c}\text { Fuel Cell } \\
\text { Generation System } \\
(\mathrm{FC} \# 1, \mathrm{FC} \# 2)\end{array}$ & 1000 & 0 & 0.0848 & 0 \\
\hline $\begin{array}{l}\text { Diesel Engine } \\
\text { Generator } \\
(\mathrm{DE} \# 1, \mathrm{DE} \# 2)\end{array}$ & 1500 & 0.3312 & 0.0156 & 0.0002484 \\
\hline $\begin{array}{c}\text { Gas Turbine } \\
\text { Generator } \\
(\mathrm{GT} \# 1, \mathrm{GT} \# 2)\end{array}$ & 1790 & 0.4969 & 0.0116 & 0.0001987 \\
\hline
\end{tabular}
represents the power sold to the utility power grid.

The procedure of the proposed MICA is show in Fig. 4.

TABLE I: PARAMETERS OF THE DGS

In using the MICA in solving the optimal schedule problem of a MG, the F\#1\&2 MG (Fig. 1) is chosen as the sample system. The correlative detailed data are first incorporated; this data include correct weather forecasting, load data, and generation data. The parameters of each DG are listed in Table I. The purchased/sold electricity cost of the F\#1\&2 MG are depicted in Fig. 5. The purchased/sold electricity cost includes peak-load, half-peak-load, and off-peak-load pricings from the upstream utility power grid during the summer season. The equivalent lumped daily load demand curves for specific summer days, which correspond to different load types at each bus, are illustrated in Fig. 6. The real power generation curves of renewable energy DG can be calculated using corresponding equations, measured solar irradiation, and wind speed data (Section II). Computation step $\Delta P$, which affects iteration number, execution time, and results, is essential to the proposed MICA. We set $\Delta P$ to $1 \mathrm{~kW}$.

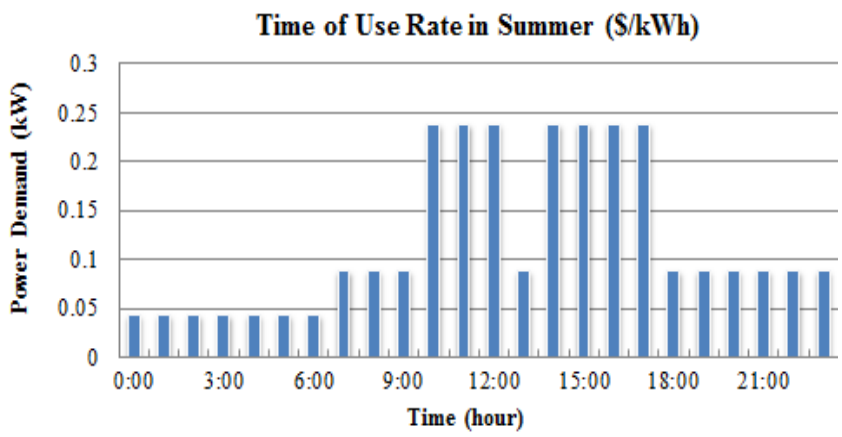

Fig. 5. Electricity purchased/sold cost of the MG.

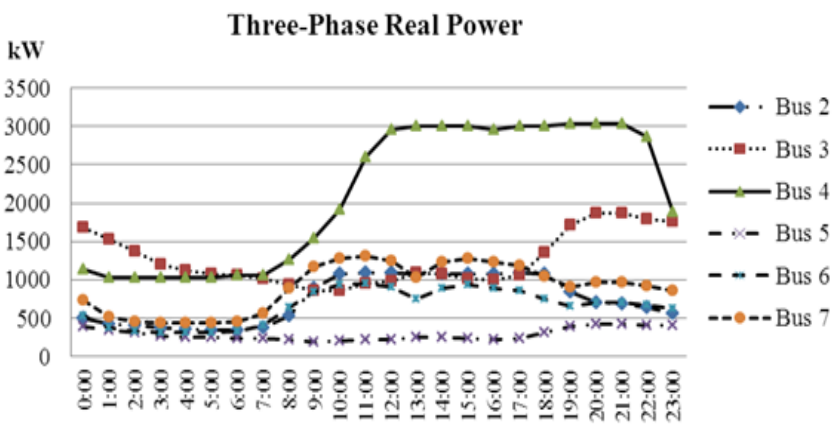

Fig. 6. Equivalent lump-load demand curve at each bus. 


\section{NUMERICAL RESULTS AND DISCUSSION}

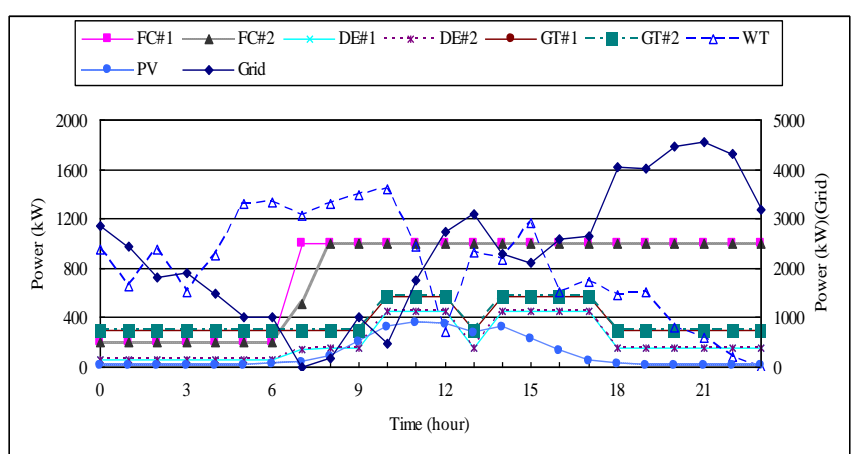

Fig. 7. Numerical results for the optimal generation of each DG and utility power grid under grid-tied operation.

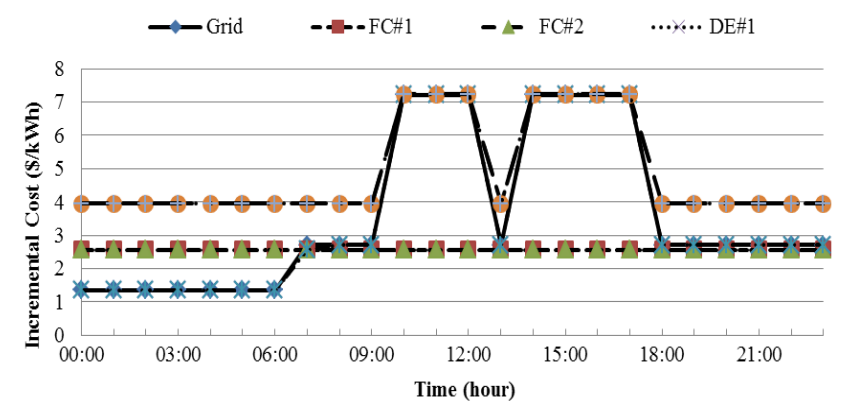

Fig. 8. Numerical results for the IC of each DG and utility power grid under grid-tied operation.

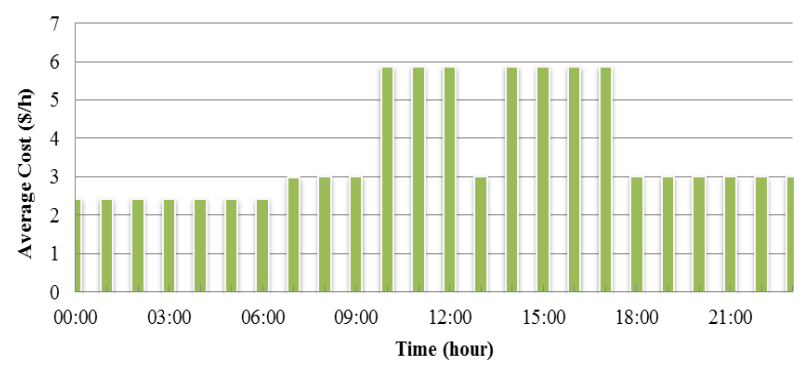

Fig. 9. Average IC of the F\#1\&2 MG under grid-tied operation.

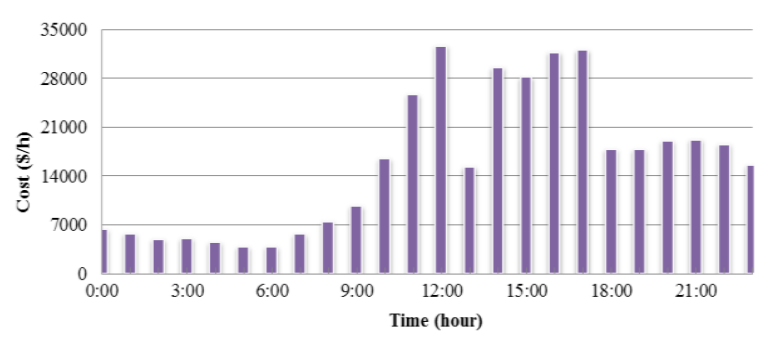

Fig. 10. Total generation cost of the F\#1\&2 MG under grid-tied operation.

On the basis of the daily load demand curve in Fig. 6 and the maximum available power generation of the renewable energy DG, we show the numerical results of the optimal schedule under the grid-tied operation of the F\#1\&2 MG (Fig. 7-Fig. 10). Fig. 7 illustrates the optimal power generation of the utility power grid and DG units under various 24-hour load demands. Fig. 8 shows the IC ( $\$$ TWD $/ \mathrm{kWh}$ ) of each DG and utility power grid. The average IC and total generation cost of the F\#1\&2 MG are shown in Fig. 9 and Fig. 10, respectively. Overall, because the electricity purchase cost from utility power grid pertains primarily to the duration of high-voltage use in summer, the equivalent electricity purchase cost per $\mathrm{kWh}$ is relatively lower than that of non-renewable energy DG. Consequently, the power output from the utility power grid is greater than those from the other DG units, except the non-fuel cost, renewable energy units. Therefore, the power generation of each unit is inversely proportional to its corresponding incremental cost; that is, as the DG incremental cost decreases, the power generated by the DG increases.

\section{CONCLUSION}

A simple and efficient MICA was proposed to solve the optimal schedule problem of a MG. Although the power levels generated by the renewable energy DG units (e.g., wind-turbine generators and photovoltaic generation systems) are inconsistent, the non-renewable energy DG units can compensate for insufficient power for load demand to maintain power balance. In terms of generation, control, and MG operation, an optimal schedule problem should be considered. Therefore, we propose an objective function that considers the minimization of fuel costs, the constraints of power generation limits, and power balance. The optimal schedule problem was also solved by the MICA program implemented using a MATLAB R2013a software package. The proposed MICA is highly suitable for solving the optimal schedule problem in MGs.

\section{ACKNOWLEDGMENT}

The authors would like to thank the National Science Council of Taiwan, R.O.C., for the financial support under Grant No. MOST 103 - 2221 - E - 018 - 020.

\section{REFERENCES}

[1] R. Lasseter, A. Akhil, and C. Marnay, "White paper on integration of distributed energy resources: The CERTSMicrogrid concept," Tech. Rep., CA, USA: Lawrence Berkeley National Laboratory, LBNL-50829, 2002.

[2] R. H. Lasseter, "MicroGrids," IEEE Power and Energy Magazine, vol. 5, no. 4, pp. 78-94, 2007.

[3] H. A. Carlos, F. P. Dulce, B. Carlos, G. Álvaro, and G. M. António, “A multi-objective evolutionary algorithm for reactive power compensation in distribution networks," Appl. Energy, vol. 86, no. 7-8, pp. 977-984, 2009.

[4] X. Gaofeng, L. Xiping, and H. Tomonori, "Stochastic unit commitment problem considering risk constraints and its improved GA-based solution method," IEEJ Transactions on Electrical and Electronic Engineering, vol. 8, no. 5, pp. 463-469, 2013.

[5] C. Audet and J. E. Jr. Dennis, "Mesh adaptive direct search algorithms for constrained optimization," SIAM Journal on Optimization, vol. 17, no. 1, pp. 188-217, 2006.

[6] A. Samui, S. R. Samantaray, and G. Panda, "Distribution system planning considering reliable feeder routing," IET Generation, Transmission \& Distribution, vol. 6, no. 6, pp. 503-514, 2012.

[7] R. Hooke and T. A. Jeeves, "Direct search solution of numerical and statistical problems," Journal of the Association for Computing Machinery, vol. 8, pp. 212-229, 1961.

[8] C. Shantanu, S. Tomonobu, Y. S. Ahmed, Y. Atsushi, and F. Toshihisa, "A fuzzy binary clustered particle swarm optimization strategy for thermal unit commitment problem with wind power integration," IEEJ Transactions on Electrical and Electronic Engineering, vol. 7, no. 5, pp. 478-486, 2012.

[9] R. Chedid, H. Akiki, and S. Rahman, "A decision support technique for the design of hybrid solar- wind power systems," IEEE Transaction on Energy Conversion, vol. 13, no. 1, pp. 76-83, 1998.

[10] M. K. C. Marwali, H. Ma, S. M. Shahidehpour, and K. H. Abdul-Rahman, "Short-term generation scheduling in photovoltaic-utility grid with battery storage," IEEE Trans. on Power Syst., vol. 13, no. 3, pp. 1057-1062, 1998. 
[11] F. Lasnier and T. G. Ang, Photovoltaic Engineering Handbook, New York: Publishing Ltd, 1990.

[12] A. J. Wood and B. F. Wollenberg, Power Generation, Operation and Control, New York: John Wiley \& Sons, Ltd, 1996.

[13] A. M. Faisal, "Microgrid modeling and online management," Ph.D. Dissertation, Helsinki University of Technology, Finland, 2008.

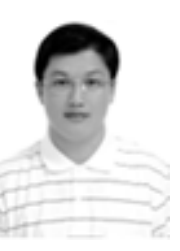

Wei-Tzer Huang was born in Taiwan, R.O.C. on August 15, 1971. He received his B.S., M.S. and Ph.D. degrees in electrical engineering from National Taiwan University of Science and Technology, Taipei, Taiwan, the Republic of China, in 1997, 1999 and 2005, respectively. Presently, he is an associate professor of National Changhua University of Education. His research interests include modeling and simulation of power systems, microgrids modeling and online management, and power electronics.
Kai-Chao Yao was born on November 4, 1971. He is a professor in the Department of Industrial Education and Technology in National Changhua University of Education. His research interests include automatic control, virtual instrument measurement, power system analysis, and technical course development.

Chun-Ching Wu was born on January 13, 1971. Presently, he is a Ph.D student in the Department of Industrial Education and Technology in National Changhua University of Education. His research interests include modeling and simulation of micro-grids. 